\title{
Diseño de un equipo de medición del desnivel en una línea ferroviaria
}

\section{Design of a measurement equipment of the unevenness in a railway line}

\author{
Kessia Carolina Ramos-Castañeda \\ Ingeniera mecatrónica \\ Universidad Santo Tomás \\ Bucaramanga, Colombia. \\ beberlihill9020010@hotmail.com \\ Jhon Jairo Gil-Peláez \\ Ph.D. Ingeniería Civil \\ Universidad Santo Tomás \\ Bucaramanga, Colombia \\ jhon_gil@hotmail.com
}

\author{
Jhon Fredy Panesso-Narváez \\ Ingeniero mecatrónico \\ Universidad Santo Tomás \\ Bucaramanga, Colombia \\ jp20033@hotmail.com \\ Reynaldo Claros-Lamus \\ Ingeniero electrónico \\ Universidad Santo Tomás \\ Bucaramanga, Colombia. \\ rclaros@hotmail.com
}

\begin{abstract}
Resumen- En este artículo se presenta el diseño de un equipo mecatrónico de medición del desnivel en una línea ferroviaria, compuesto por tres fases. La primera de ellas consiste en un sistema mecánico que le permite desplazarse sobre las vías férreas, para el cual se desarrolla un análisis dinámico y se realiza un modelamiento estructural utilizando la técnica de elementos finitos y el software Solidworks. La segunda, es un sistema de adquisición de datos que comprende todo el diseño electrónico del dispositivo, la selección de los acelerómetros idóneos para la aplicación y el uso de un microcontrolador de la familia Microchip para la recepción y procesamiento de la información obtenida de los sensores. La última, es la etapa de visualización, en la que se usa una LCD para mostrar los datos de forma local y a través de un módulo XBee se envían a un punto de control remoto con el fin de monitorear a través de una interfaz realizada en LabVIEW. Este sistema busca mejorar y optimizar la detección de desniveles, crear facilidad de visualización en situaciones de poca o nula visibilidad, reducir el personal involucrado en la toma de mediciones manuales y brindar información oportuna.
\end{abstract}

Palabras clave- Desnivel, línea ferroviaria, medición.

Abstract- This article presents the design of a mechatronic unevenness measurement equipment on a railway line, consisting of three phase. The first one is a mechanical system that allows you to go on the railways, for which develops a dynamic analysis and structural modeling using the finite element technique and the SolidWorks software. The second is a data acquisition system comprising all the device's electronic design, the selection of accelerometers suitable for the application and use of a microcontroller of the Microchip family for receiving and processing information from sensors. The last one is the visualization stage, in which an LCD is used to display data locally and through an XBee module is sent to a remote control point in order to moni- tor via an interface made in LabVIEW. This system aims to improve and optimize the detection of unevenness, creating easy viewing in low or no visibility, reduce staff involved in making manual measurements and provide timely information.

Keywords- Unevenness, railway line, measuring.

\section{INTRODUCCIÓN}

En las líneas ferroviarias a nivel mundial se monitorean parámetros como el nivel, ancho y alineación de la vía, la curvatura, el perfil del carril, entre otros [1], con el fin de detectar problemas como el desgaste de los rieles, fisuras, roturas, hundimientos en la topografía del terreno [2], reducir el riesgo de accidentes, garantizar la seguridad de los operarios y el bienestar de la carga [3].

Considerando que el nivel es uno de los parámetros más importantes en el momento de realizar el monitoreo [4], se ha encontrado que existen dos formas de hacer su medición: manual y automática [5]. En la primera se trata de herramientas manuales con las que no se registran continuamente los datos medidos. Son equipos de manejo manual como unidades de topografía, regla de ancho y peraltes, asas de flechar y galgas [5], [6] que un operario utiliza desplazándose sobre la vía entre la compactadora y la reguladora [7], lo que representa un escenario de riesgo, ya que se encuentra expuesto en la línea de acción de los equipos. En la segunda se emplean equipos 
que van sobre el riel, con un sistema de sensores que toman y registran la medida constantemente; además, suelen ir equipados con sistemas auxiliares de posicionamiento por satélite, sistemas informáticos y elementos electrónicos que permiten la transmisión de datos a puntos remotos, entre otros tipos de tecnologías [5].

Teniendo en cuenta la importancia de la medición del nivel en las líneas ferroviarias se han desarrollado diversos dispositivos que permiten realizar el proceso de forma automática. En la Universidad de Nihon en Japón se elaboró un sistema de protección automática para un tren, en el que se desarrolló un novedoso método de medición [8]. En el 2008 se diseñó y construyó una unidad móvil para la detección de desnivel en líneas ferroviarias en Japón, compuesto por un ADC NI USB 6009 acelerómetros, un micro PC Sony VGN-UX50 y un GPS [9]. Posteriormente se elaboró un sistema de medición que utilizaba láseres, cámaras y herramientas inerciales ubicadas directamente en el tren [4]. También se construyó en Rusia un sistema de diagnóstico, que comprendía un sistema mecánico-estructural, sobre el cual se ubicaban elementos electrónicos, incluyendo una PC, para que el operario tuviese acceso a los datos medidos y pudiese generar reportes y alarmas en caso de ser necesario [3]. Finalmente se fabricó un sistema de diagnóstico ferroviario en que se empleaban sensores MEM (microelectromechanical) y se puso en práctica con éxito en los ferrocarriles de Rusia [10].

A nivel nacional la medición se realiza de forma manual, ya que la adquisición de sistemas automáticos comerciales es costosa, de manera que actualmente se hace usando carros manuales de auscultación, así que no se tiene una medición y registro constante del nivel de una línea ferroviaria [5], por tal razón este artículo presenta el diseño de un dispositivo automático de bajo costo para la medición del desnivel, compuesto por un sistema mecánico, uno de adquisición y otro de visualización. El primero comprende el análisis dinámico del prototipo y de las ruedas, el diseño de los ejes, la selección de los rodamientos y el modelamiento por elementos finitos con el Software Solidworks. El segundo corresponde al diseño electrónico, en el que se seleccionan los acelerómetros, el microcontrolador, progra- mado en PIC C compiler y se realiza la simulación en Proteus. En el tercero se muestran los datos - alertas de forma local en una LCD y de manera remota a través de un XBee y logra visualizarse en una interfaz realizada en LabVIEW. Por último, todo el sistema se simula y se verifica su funcionamiento.

\section{CONSIDERACIONES GENERALES $Y$ METODOLOGÍA}

En general, para una buena adquisición de datos en un sistema instrumentado, se tienen en cuenta aspectos como: la técnica adecuada para la toma de la medición, la repetitividad, la observabilidad, el uso apropiado de un instrumento de medición, la calibración, la facilidad de visualización, la precisión y el no exponer al personal en el desarrollo de actividades en escenarios, bajo evidentes exposiciones de riesgo. El proceso actual de medición no obedece a los aspectos mencionados con anterioridad, por lo tanto, es necesario llevar a cabo el planteamiento de una solución efectiva a la problemática existente que permita la toma de decisiones en el momento preciso.

Para el desarrollo de este proyecto se aplica la metodología de la Estructura Jerárquica de Producto (PBS, Product Breakdown Structure) [11], [12], la cual es una herramienta de gestión de proyectos que describe el objetivo del mismo y sus dimensiones, de lo general a lo particular.

El diseño del equipo de medición propuesto incluye a su vez tres sistemas, el Sistema Mecánico, el Sistema de Adquisición y el Sistema de Visualización.

El Sistema Mecánico contiene los requerimientos necesarios para garantizar el funcionamiento del equipo de medición, en él se contempla el diseño y selección de partes funcionales del sistema, tales como, las partes móviles y el chasis, como se muestra en la Fig. 1a. Para el desarrollo del sistema mecánico, se realizaron los análisis dinámicos requeridos, para lo cual se consideraron las condiciones críticas. Después se realizó un prediseño y las simulaciones respectivas, mediante el modelamiento con elementos finitos, utilizando un software comercial, para posteriormente evaluar los resultados obtenidos y hacer los ajustes en el diseño que se consideren necesarios. 
Para el Sistema de Adquisición, se realizaron las siguientes actividades: la adquisición de datos y la alimentación de energía, como se puede observar en la Fig. $1 b$.

En la etapa de visualización se indica el desnivel de forma local en una pantalla LCD, que también suministra información complementaria, como es la identificación del riel que presenta una mayor inclinación; la visualización de alarmas y una interfaz para supervisar el proceso de forma remota, como se puede observar en la Fig. 1c.

Fig. 1. RAMIFICACIÓN A. SISTEMA MECÁNICO B. SISTEMA DE ADQUISICIÓN DE DATOS C. SISTEMA DE VISUALIZACIÓN
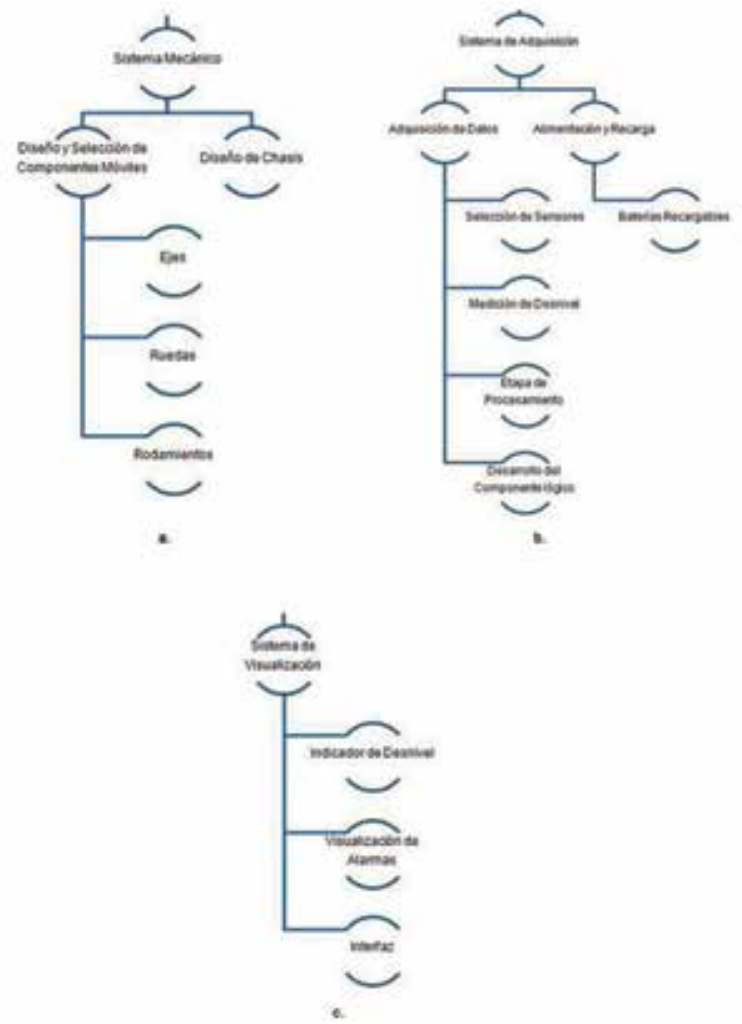

Fuente: autores.

A continuación se presentan las diferentes actividades desarrolladas en cada uno de los tres sistemas mencionados anteriormente.

\section{SISTEMA MECÁNICO}

El diseño de la plataforma mecánica del equipo se lleva a cabo teniendo en cuenta las siguientes consideraciones: el dispositivo se desplaza sobre la vía férrea guiado por la Compactadora Jackson
Modelo 6700. Para estimar la fuerza impulsora necesaria para el movimiento del prototipo, se analiza el caso más crítico, el cual se presenta cuando el desplazamiento se realiza en un plano inclinado a $45^{\circ}$. Los cálculos se realizan considerando que el prototipo se desplaza a una velocidad típica de operación de $10 \mathrm{Km} / \mathrm{h}$. $(2.7 \mathrm{~m} / \mathrm{s}) \mathrm{la}$ cual se alcanza en un tiempo de 10 segundos.

Para realizar el análisis dinámico del sistema, se consideran los siguientes pesos: Peso sin sistema de medición: $30 \mathrm{kgf}(0.29 \mathrm{kN})$ y Peso máximo: 50 kgf $(0.49 \mathrm{kN})$.

\subsection{Análisis dinámico del prototipo}

Las fuerzas que actúan sobre el prototipo se muestran en el Diagrama de Cuerpo Libre (DCL), presentado en la Fig. 2. En este análisis se considera el plano inclinado a un ángulo $\theta$ de $45^{\circ}$.

Fig. 2. DIAGRAMA DE CUERPO LIBRE DEL MODELO

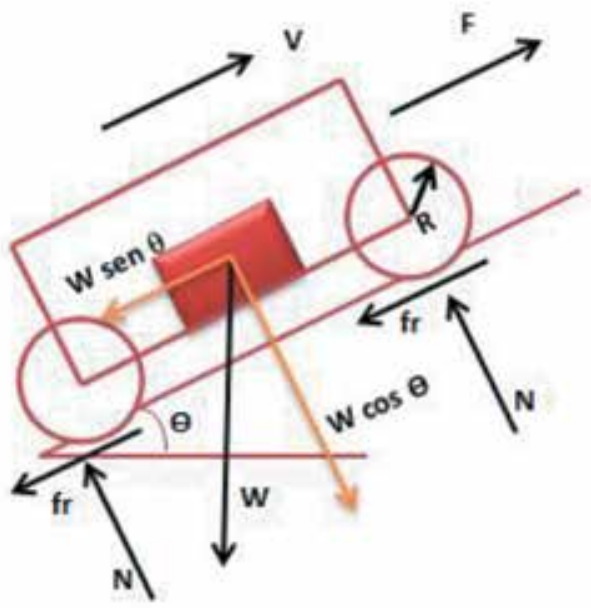

Fuente: autores.

donde, fr = Fuerza de Fricción, $(\mathrm{N}), \mathrm{N}=$ Fuerza Normal, (N), W = Peso del equipo, (N), V = Velocidad del equipo, $(\mathrm{m} / \mathrm{s})$ y $\mathrm{F}=$ Fuerza necesaria para generar desplazamiento, $(\mathrm{N})$.

Una vez se identifican las fuerzas que actúan sobre el modelo propuesto, se plantean las sumatorias de fuerzas en los ejes $\mathrm{x}$ y $\mathrm{y}$.

$$
\begin{gathered}
\Sigma F_{x}=-4 \cdot f_{r}-W \cdot \operatorname{sen} \theta+F=m \\
\Sigma F_{y}=-4 \cdot N-W \cdot \cos \theta=0
\end{gathered}
$$

Para el cálculo del coeficiente de fricción se tiene, 


$$
f_{r}=\mu \mathrm{k} \cdot N
$$

Teniendo en cuenta que los elementos en contacto son de acero, el coeficiente de fricción cinética se toma como $\mu \mathrm{k}=0.57$, el cual corresponde Acero - Acero [13], con lo cual se obtiene $\mathrm{fr}=49.407 \mathrm{~N}$

Asumiendo un movimiento uniformemente acelerado se cumple la siguiente ecuación:

$$
V_{f}=\mathrm{V}_{0}+a \cdot t
$$

donde Vf y Vo son las velocidades final e inicial, respectivamente. A partir de la cual se procede al cálculo de la aceleración,

$$
a=0.27 \frac{m}{s^{2}}
$$

De la sumatoria de fuerzas en el eje $\mathrm{x}$, la cual se muestra en (1) [14], se despeja el valor de la fuerza F:

$$
F=m \cdot a+f_{r}+W \cdot \sin \theta(5)
$$

Se concluye así que la fuerza necesaria para generar movimiento en el equipo de medición de desnivel es $\mathrm{F}=409.625 \mathrm{~N}$

\subsection{Análisis dinámico de las ruedas}

Las fuerzas que actúan sobre la rueda se muestran en el DCL presentado en la Fig. 3.

Fig. 3. DIAGRAMA DE CUERPO LIBRE DE LA RUEDA

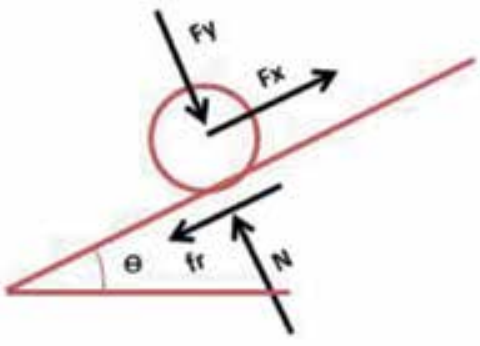

Fuente: autores

donde, $\mathrm{Fx}=$ Componente en el eje $\mathrm{x}$, de reacción generada por la rueda en el eje, $(\mathrm{N}), \mathrm{Fy}=$ Componente en el eje y, de reacción generada por la rueda en el eje, $(\mathrm{N}), \mathrm{fr}=$ Fuerza de Fricción, $(\mathrm{N})$, $\mathrm{N}=$ Fuerza Normal, (N). De donde se obtienen las reacciones en las llantas:

$$
\mathrm{Fx}=49.407 \mathrm{~N} \quad \mathrm{Fy}=86.679 \mathrm{~N}
$$

\subsection{Fuerzas en los ejes}

Las fuerzas que actúan sobre los ejes se muestran en el DCL de la Fig. 4.

Fig. 4. DIAGRAMA DE CUERPO LIBRE DEL EJE

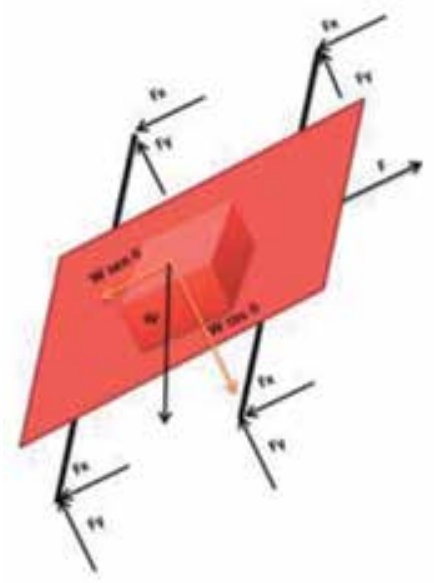

Fuente: autores.

Las fuerzas Fx y Fy son las reacciones generadas por las ruedas en los ejes, las cuales se calcularon en el punto anterior:

$$
\mathrm{Fx}=49.407 \mathrm{~N} \quad \mathrm{Fy}=86.679 \mathrm{~N}
$$

Calculando la resultante de estas reacciones se obtiene la fuerza puntual en el eje igual a:

$$
\mathrm{P}=99.772 \mathrm{~N}
$$

\subsection{Diseño del eje tubular}

Para el diseño del eje se considera una sección tubular y se asume una con longitud de $30 \mathrm{~cm}$ y un radio exterior de $1.5 \mathrm{~cm}$, la cual, debido a simulaciones mecánicas, es geométricamente resistente a la carga estimada y corresponde a un perfil comercial. La condición de carga crítica se presenta cuando el equipo se encuentra en un plano inclinado a $45^{\circ}$, siendo en este caso la carga puntual $\mathrm{P}$ de $99.77 \mathrm{~N}$. El eje se modela como una viga en voladizo, como se muestra en la Fig. 5.

Fig. 5. EJE TUBULAR

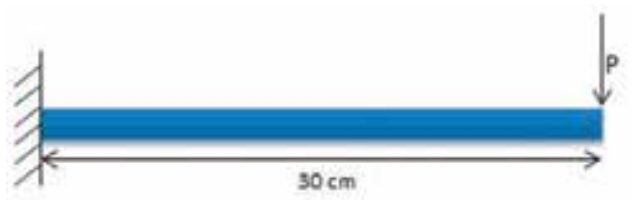

Fuente: autores.

Teniendo en cuenta estas dimensiones del eje, se continúa con la verificación de esfuerzos. Se 
calcula el momento producido por la carga puntual en el apoyo [15],

$$
M=P \cdot L_{a}
$$

donde, $\mathrm{P}=$ Carga puntual en el eje, (N). $\mathrm{L}_{a}=$ Longitud de la viga, (m).

De la ecuación (6), se obtiene que el valor del momento es de $29.932 \mathrm{~N} \cdot \mathrm{m}$.

Tomando la sección transversal del eje, se encuentra el esfuerzo normal [16] $\sigma$, producido por el momento flector, el cual es igual a:

$$
\sigma=\frac{M \cdot r_{\text {ext }}}{I}(7)
$$

donde, $\mathrm{M}=$ Momento por carga Puntual en el apoyo, $(\mathrm{N}), \mathrm{r}_{\text {ext }}$ = Radio del eje, $(\mathrm{m}), \mathrm{I}=$ Momento de inercia de la sección transversal, $\left(\mathrm{m}^{4}\right)$.

De la ecuación (7), se obtiene el esfuerzo normal igual a, $\sigma=32.835 \mathrm{MPa}$

La ecuación (8), se emplea para calcular el esfuerzo cortante [17],

$$
\tau=\frac{V_{a} \cdot Q}{I \cdot e}
$$

donde, $\tau=$ Esfuerzo Cortante, $\left(\mathrm{N} / \mathrm{m}^{2}\right)$, Va= Fuerza Cortante, $(\mathrm{N}), \mathrm{Q}=$ Primer momento de área, $\left(\mathrm{m}^{3}\right)$.

$\mathrm{I}=$ Momento de Inercia, $\left(\mathrm{m}^{4}\right), \mathrm{e}=$ Espesor, $(\mathrm{m})$.

Reemplazando, se obtiene, $\tau=1.483 \mathrm{MP} a$

De acuerdo con los esfuerzos calculados anteriormente, el desplazamiento máximo en el extremo libre del eje es igual a, $\delta_{\text {máx }}=0.952 \mathrm{~mm}$, siendo el desplazamiento admisible [18], $\delta_{c}=1.389 \mathrm{~mm}$

Por lo tanto, el desplazamiento máximo es menor que el desplazamiento admisible. A partir de lo anterior, se concluye que la geometría seleccionada para el eje cumple los requerimientos de esfuerzo y de rigidez.

\subsection{Ruedas}

Las ruedas son diseñadas en acero [19], dado que estarán en operación permanente con los rieles. Para la dureza se considera la misma del riel, siendo la dureza interna $341 \mathrm{HB}[20]$ o superior. De acuerdo con las dimensiones del eje, y teniendo en cuenta las proporciones del modelo, se proponen las siguientes dimensiones: Diámetro externo: 140mm, Diámetro Interno: 80mm, Diámetro del eje: $30 \mathrm{~mm}$ y Espesor rueda: $80 \mathrm{~mm}$

\section{6 Selección de rodamientos}

Las ruedas se instalaron sobre rodamientos, los cuales se seleccionaron teniendo en cuenta las capacidades de carga y los diámetros deben soportar carga radial, por lo tanto, los rodamientos adecuados son los de bolas [21], apropiados para cargas radiales y exigen muy poco mantenimiento. Las cargas presentes en el rodamiento son: $F_{x}=49.407 N$ y $F_{y}=86.679 N$. La carga radial presente en el rodamiento es: $\mathrm{F}_{\mathrm{r}}=90.771 \mathrm{~N}$

Conociendo las cargas y tomando como base fundamental el diámetro del eje, se realiza la selección del rodamiento en el catálogo de producto de SKF (Svenska Kullagerfabriken). Se selecciona el rodamiento de Bolas de referencia 62306-2RS1 [22].

\subsection{Simulaciones del sistema mecánico}

Basados en los resultados obtenidos del diseño mecánico, se realiza la simulación por tensión, desplazamiento y factor de seguridad de cada uno de los componentes del equipo, por medio de la teoría de Elementos Finitos [23] y el software SolidWorks [24] [25], como se muestra en la Fig. 6.

Fig. 6. RESULTADOS DE SIMULACIONES POR TENSIÓN Y DESPLAZAMIENTO
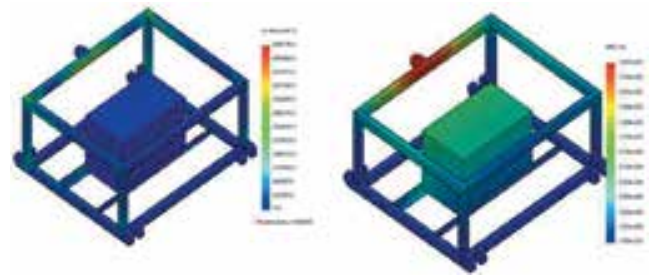

Fuente: autores.

Los resultados obtenidos muestran que los elementos diseñados fueron bien estimados, y las geometrías y materiales seleccionados son aptos para la aplicación. El ensamble final del sistema mecánico se muestra en la Fig. 7.

Fig. 7. ENSAMBLE SISTEMA MECÁNICO

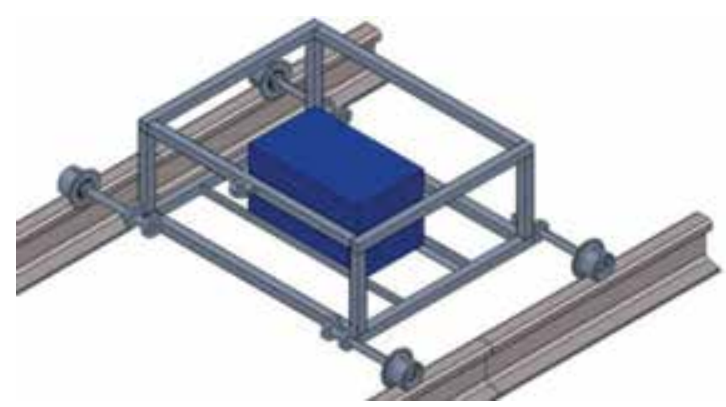

Fuente: autores. 


\section{SISTEMA DE ADQUISICIÓN DE DATOS}

El diseño electrónico del modelo se lleva a cabo teniendo en cuenta las siguientes consideraciones: La medición de desnivel se realiza cada 2 segundos y el prototipo se desplazará a una velocidad típica de operación de $10 \mathrm{Km} / \mathrm{h}$, la cual se alcanzará en un tiempo de 10 segundos. A continuación se detallan las fases que componen el sistema de adquisición de datos.

\subsection{Adquisición de datos}

Teniendo en cuenta que se requiere realizar la medición del desnivel en las vías férreas y conociendo el sistema por instrumentar, y el empleo de acelerómetros en otras aplicaciones similares [9], se inicia la búsqueda de estos, que son sensores utilizados en la medición de la inclinación. EI MMA7260QT [26] es un acelerómetro capacitivo, su principio de funcionamiento se basa en el cambio de la capacitancia del elemento interno en proporción a la aceleración aplicada. Este acelerómetro consiste en una celda sensitiva semiconductora, que mide la aceleración como la diferencia entre capacitancias de dos placas fijas y una placa central móvil, la cual presenta variación de su posición cuando el sensor es sometido a una aceleración.

\section{2 Salidas del sensor}

La ecuación (9), que rige el funcionamiento del sistema muestra que el valor de tensión en cada una de las salidas será igual a la mitad del valor de alimentación (Offset), más un término que dependerá de la aceleración registrada [27]. Es decir:

$$
\text { Salida }_{x, y, z}=\frac{V_{s}}{2} \pm F \cdot A
$$

donde, $\mathrm{V}_{\mathrm{s}}$ : tensión de alimentación, (V) y F·A: factor de aceleración, $\left(\mathrm{m} / \mathrm{s}^{2}\right)$. Este factor de aceleración podrá sumar o restar si la aceleración detectada es positiva o negativa.

\subsection{Medición de desnivel}

Para efectos de medición del desnivel se precisa la salida del eje $\mathrm{x}$, teniendo en cuenta que el eje z se encuentra ortogonal se anula la componente de este eje, finalmente se obtiene:

$$
X_{\text {out }}=\frac{V_{s}}{2} \pm S \cdot \sin \gamma
$$

donde, $X_{\text {out }}=$ Respuesta del sensor con respecto al eje $\mathrm{X},(\mathrm{V}), \mathrm{S}=$ Sensibilidad, (V/g) y $\gamma=$ Ángulo que forma el eje $\mathrm{z}$ respecto al sensor.

Se tienen en cuenta dos casos, el primero donde el desnivel en el riel derecho sea mayor que en el riel izquierdo y, el segundo, donde el desnivel en el riel izquierdo sea mayor que en el riel derecho. Para ambos casos se emplea el primer término de la ecuación (10), la variación se presenta en el factor de aceleración, para el primer caso, este factor es positivo, y para el segundo es negativo.

\subsection{Etapa de procesamiento}

En esta fase se realiza la recepción y procesamiento de datos, para lo cual se emplea el PIC18F4550, de tal manera que la señal de voltaje recibida se convierte a desnivel, se establece la comunicación con el modulo LCD para la visualización de los datos y alerta visuales en modo local y se transmiten por comunicación serial para su posterior procesamiento en el mando remoto a través del PC y el software LabVIEW, como se muestra en la Fig. 8.

Fig. 8. DIAGRAMA DE BLOQUES DE ETAPA DE PROCESAMIENTO EN EL
MICROCONTROLADOR

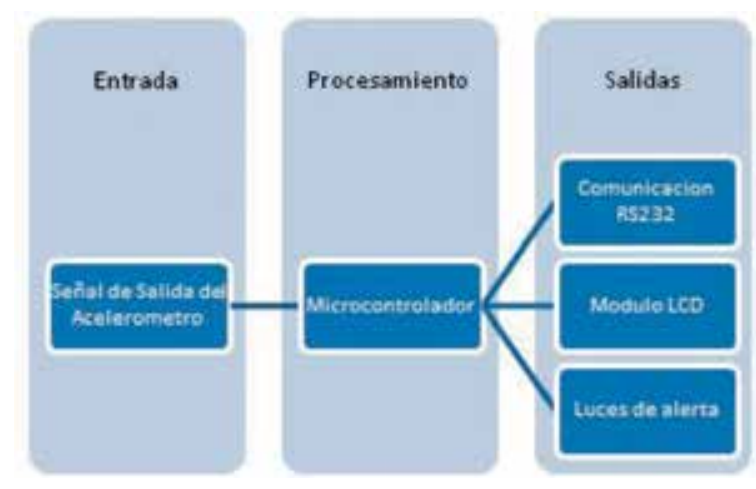

Fuente: autores.

\subsection{Diseño y desarrollo del componente lógico o software}

La programación se implementó en lenguaje C, en el software PIC C Compiler y las simulaciones se realizaron en Proteus. El programa consta de cinco etapas, que se muestran a continuación: 
- Primera etapa. Se tienen las funciones principales y a su vez las librerías necesarias para el correcto funcionamiento del PIC 18F4550 [28]

- Segunda etapa. Corresponde a la lectura de las señales análogas, que entrega el acelerómetro como salida en forma de voltaje.

- Tercera etapa. Consta del cálculo matemático donde se convierte la señal de voltaje a valores de desnivel.

- Cuarta etapa. Consiste en saber a qué nivel de tolerancia corresponde el valor anteriormente calculado, teniendo en cuenta que se manejan tres niveles de tolerancias $(a, b, c)$ y fuera de tolerancia.

- Quinta etapa. Por último, se transmite el valor de desnivel por medio de comunicación RS 232, además se encienden los indicadores locales de desnivel y se transmite el dato al módulo LCD para su visualización.

\subsection{Alimentación y recarga}

El sistema de alimentación de la plataforma consta de una batería, la cual se selecciona realizando el cálculo del consumo máximo de corriente equivalente a $0.8 \mathrm{~A}$, aproximadamente, y dado que la batería puede entregar $12 \mathrm{~A} / \mathrm{h}$ (Amperios hora) el prototipo puede alcanzar un funcionamiento continuo de 15 horas. Se seleccionó una batería que provee horas de operación adicionales, teniendo en cuenta que el prototipo estará operando por turnos de mantenimiento de 8 horas, los cuales se realizan en zona rural, razón por la que no es fácil encontrar una fuente de energía.

\subsection{Acople de sistema electrónico en el equipo}

En la Fig. 9 se muestran los elementos electrónicos por separado y finalmente en la Fig. 10 se muestra cómo se encuentra cada uno de los elementos electrónicos acoplados en el ensamble final del equipo.
Fig. 9. DETALLE DE LOS ELEMENTOS ELECTRÓNICOS

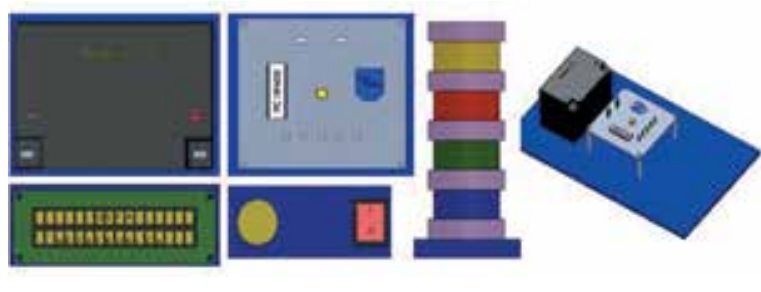

Fuente: autores.

Fig. 10. SISTEMA DE ADQUISICIÓN DE DATOS ACOPLADO AL EQUIPO

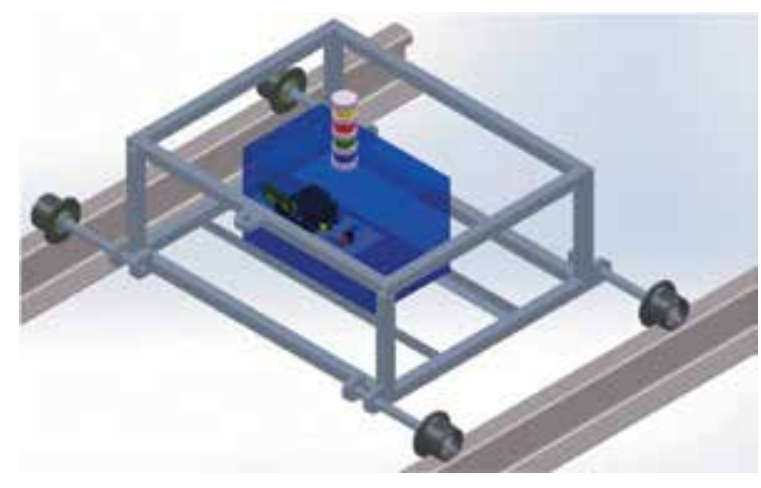

Fuente: autores.

\section{SISTEMA DE VISUALIZACIÓN}

Este módulo es el encargado de recibir los valores del desnivel que son calculados por el microcontrolador, basado en la señal que recibe del acelerómetro, posteriormente se muestran en una LCD de forma local, luego son enviados por el Xbee [29], y recibidos en una interfaz de comunicación desarrollada en LabVIEW, que permite la visualización y control del desnivel.

En la Interfaz LabVIEW se recibe la señal de comunicación serial a través del puerto RS 232, se procesa y se realiza la etapa de visualización del comportamiento de variables y alarmas de acuerdo con el nivel de tolerancia, la aplicación almacena los valores de medición, teniendo en cuenta que al detectar un valor del desnivel mayor al máximo permisible se realiza el envío de correos electrónicos al supervisor, como se muestra en la Fig. 11.

\section{SIMULACIÓN}

Basados en el desarrollo de la programación, los circuitos electrónicos expuestos anteriormente y la interfaz de visualización, se realizó la si- 
mulación electrónica del sistema, implementando el programa Virtual Serial Port, el cual permite la simulación del envío y recepción de datos, que se propone realizar mediante el Xbee. La señal de salida de voltaje del acelerómetro se simula como una resistencia variable. A continuación se muestra la simulación, la cual consiste en generar desde Proteus una señal electrónica, que muestra un desnivel de Tolerancia Tipo a, donde el Riel izquierdo se encuentra más alto que el Riel derecho, esta señal se envía por el puerto serial.

En la Interfaz de monitoreo se recibe la señal simulada y enviada por el puerto señal en la etapa anterior, y se visualiza el comportamiento en el tiempo del desnivel, Nos indica el nivel de tolerancia, esta información se envía por correo electrónico al supervisor encargado, en forma de reporte, el cual complementa la herramienta, dándole valor agregado, como se muestra en la Fig. 12.

Fig. 11. DIAGRAMA DE BLOQUES DE ETAPA DE VISUALIZACIÓN EN LABVIEW

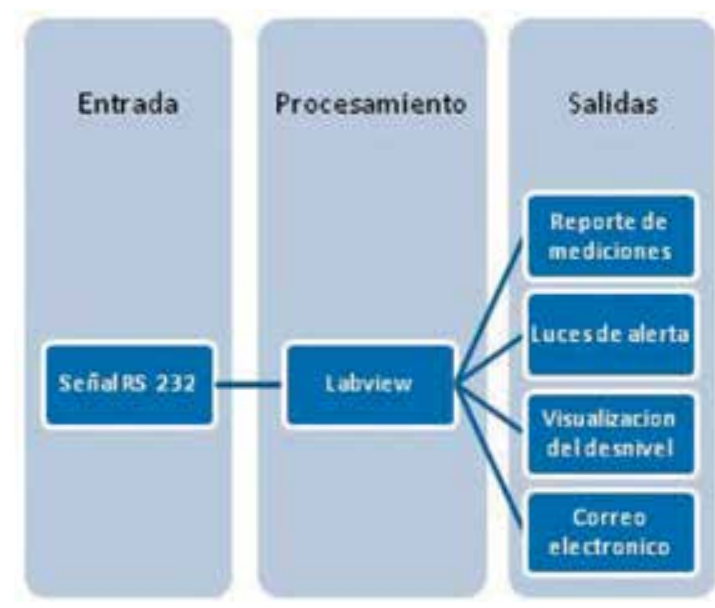

Fuente: autores.

Fig. 12. SIMULACIÓN EN LABVIEW

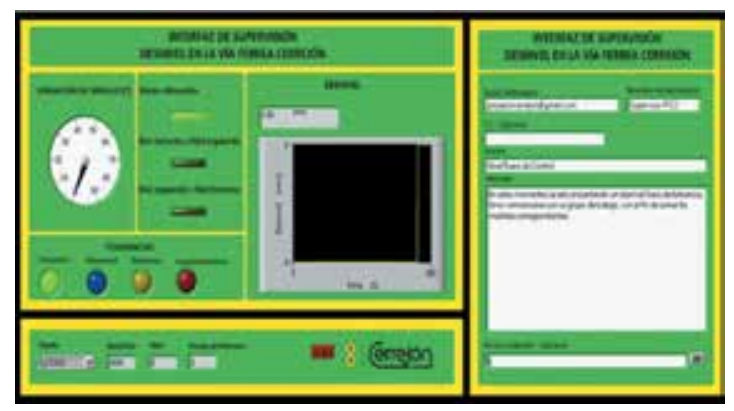

Fuente: autores.
Luego de varias simulaciones, se obtiene como resultado, la posibilidad de simular el envió y recepción de datos y la visualización de los diferentes niveles de alarma, tanto de forma local como remota. La evaluación de los resultados obtenidos se expone en la Tabla I.

TABLA I

RESULTADOS DE SIMULACIÓN

\begin{tabular}{|l|c|}
\hline \multicolumn{1}{|c|}{ Característica } & Funcionamiento \\
\hline Circuito electrónico & $\checkmark$ \\
\hline Programación & $\checkmark$ \\
\hline SCADA & $\checkmark$ \\
\hline Envió y recepción de datos & $\checkmark$ \\
\hline Detección de desnivel & $\checkmark$ \\
\hline $\begin{array}{l}\text { Visualización de los diferentes niveles de } \\
\text { alarma }\end{array}$ & $\checkmark$ \\
\hline Envío de correo electrónico & $\checkmark$ \\
\hline
\end{tabular}

\section{CONCLUSIONES}

Se diseñó un Equipo de Medición Mecatrónico de desnivel guiado por una plataforma mecánica que se desplaza sobre las vías férreas, para ser utilizado como herramienta de soporte en su mantenimiento.

El diseño mecánico se realizó partiendo de un prediseño, obtenido a partir de la teoría clásica de resistencia de materiales. Las simulaciones con elementos finitos realizadas en Solidworks mostraron que la estructura propuesta como diseño final satisface los requerimientos de carga del equipo.

El equipo propuesto mejora significativamente la seguridad del proceso de medición del desnivel en las vías férreas, salvaguardando la integridad física de los operarios.

El equipo diseñado ayuda a reducir los tiempos muertos por el manteniendo en las vías, ofrece una mayor confiabilidad y repetitividad de la medición, así como la posibilidad de comparar la medición permanente. Esto genera una mayor confianza para la toma de decisiones y posibilita la aplicación de la acción correctiva de forma inmediata, en el mismo momento en que se identifica el desnivel.

Los elementos utilizados para el desarrollo del dispositivo son de bajo costo, lo que lleva a que el sistema pueda ser implementado y adquirido por empresas que requieran el constante monitoreo y mantenimiento de líneas ferroviarias, tanto a nivel nacional como internacional. 


\section{REFERENCIAS}

[1] CEN - CENELEC, "Railway applications - Track - Track geometry quality - Part 2: Measuring systems - Track recording vehicles," NSAI Standars, Irish Standard I.S.EN 13848-6, Europa, 2014.

[2] B. Peña, K. Sánchez, R. Teixeira, R. Veliz y Y. Alayon, Mantenimiento geométrico de la vía férrea del tramo Caracas - Tuy medio, Caracas, Venezuela: Universidad Nacional Experimental Politécnica de la Fuerza Armada, 2008.

[3] E. D. Bokhman, A. M. Boronachin, Y. V. Filatov, D. Y. Larionov, L. N. Podgornaya, R. V. Shalymov y G. N. Zuzev, "Optical - inertial system for railway track diagnostics," in Inertial Sensors and Systems Symposium (ISS), Karlsruhe, Germany, 2014.

[4] S. S. Kim, C. Park, Y. G. Kim y C. Park, "Parameter characteristics of rail inspection measurement system of HSR-350x," Journal of Mechanical Science and Technology, n² 23, pp. 1019-1022, 2009.

[5] Viceministerio de Infraestructura, "Manual de normatividad férrea Parte II: Definición de aspectos técnicos de mantenimiento y seguridad ferroviaria," Ministerio de Transporte, Colombia, 2013.

[6] Empresa Portuaria Arica, EPA , "Proyecto de remediación de la vía férrea del ferrocarril Arica La Paz," 2009. http://www.puertoarica.cl/www/contenidos/puerto_arica/licitacion_EFE_EPA_200901/BASES_TECNICAS_PROYECTO_REMEDIACION_VIA_FERREA_FERROCARRIL_ARICA_LA_PAZ.pdf.

[7] Track Speq, Soluciones Ferroviarias, «Equipo ferroviario," 2015. http://trackspeq.com/es/equipo/.

[8] S. Takahashi, H. Mochizuki y H. Nakamura, "Development of measurement technology on probe train for signaling equipment," de The Institution of Engineering and Technology International Conference on Railway Condition Monitoring, Birmingham, Reino Unido, 2006.

[9] Y. Mizuno, Y. Fujino, K. Kataoka y Y. Matsumoto, «Development of a mobile sensing unit and its prototype implementation," Tsinghua Science and Technology, vol. 13, n S1, pp. 223-227, 2008.

[10] R. V. Shalymov, L. N. Podgornaya, D. Y. Larionov, Y. V. Filatov y A. M. Boronahin, "Measurement system for railway track condition monitoring," de IEEE Young Researchers in Electrical and Electronic Engineering Conference (EIConRusNW), Petersburg, Rusia, 2015.

[11] P.B.S., "Product breakdown structure concept," 2014. [En línea]. Available: ‘www.productbreakdownstructure.com.

[12] H. Yongping, Z. Penfgei y S. Weiping, "Study of multiagent task model based on product structure breakdown," in Fifth International Conference on Information Technology: New Generations, Las Vegas, Estados Unidos, 2008.
[13] R. Serway y J. Jewett, "Coeficientes de fricción," in Física Universitaria, New York, McGraw-Hill, 2009, pp. 69-72.

[14] A. Bedford, "Fuerza, masa y aceleración,» in Dinámica: Mecánica para ingeniería, México, Pearson, 2000, pp. 101-137.

[15] P. F. Beer y E. R. Johnston, "Momento Flector," in Mecánica de materiales, Bogotá D.C., Mc Graw Hil, 1993, pp. 75-83.

[16] E. J. Bowles , "Cálculos de Esfuerzos," in Diseño de Acero estructural, México D.F., LIMUSA Noriega Editores, 1996, pp. 105-122.

[17] G. Budynas, R. Shigley y J. K. Nisbett, "Esfuerzos en Ejes," en Diseño en Ingeniería Mecánica, México, Mc Graw Hill, 2008, pp. 95-103.

[18] E. J. Bowles, "Desplazamientos en ejes," en Diseño de acero estructural, México D.F., LIMUSA Noriega Editores, 2006, pp. 230-245.

[19] C. Lonsdale, S. Dedmon y J. Pilch, "Recent developments in forged railroad wheels for improved performance," in Proceedings of the ASME/IEEE Joint Rail Conference, Colorado, USA, 2005.

[20] Arema, Especificaciones de vías férreas trocho ancha' en Ene. 2001., México: AREMA Reglamento Del Servicio Ferroviario, 2001.

[21] V. Badiola, "Rodamientos," en Diseño de Máquinas I, Navarra, España, Universidad de Navarra, 2004, pp. 93-116.

[22] SKF, "Rodamiento de bolas," en Catalogo SKF, 2010, pp. 22-26.

[23] O. C. Zienkiewics y R. L. Taylor, El método de los elementos finitos, España: McGrawHill, 1994.

[24] S. González Gómez, El gran libro de Solidworks, España: Marcombo, S.A., 2014.

[25] S. González Gómez, Solidworks simulation, España: Ra-Ma, 2010.

[26] Freescale, "MMA7260QT," in Technical Data Freescale Semiconductor, 2008, p. 4.

[27] K. Ogata, "Analisis de Sistemas de Control," en Dinamica de Sistemas, México, Prentice Hall, 1987, pp. 494-523.

[28] Microchip, «PIC 18F4550,» in Technical Data Micopchip, 2009, pp. 2-4.

[29] Zigbee, "XBee,» in Technical data zigbee, 2011, pp. 1-3. 